CORRECTION

https://doi.org/10.1038/s41586-018-0689-7

\title{
Publisher Correction: Functional aspects of meningeal lymphatics in ageing and Alzheimer's disease
}

Sandro Da Mesquita, Antoine Louveau, Andrea Vaccari, Igor Smirnov, R. Chase Cornelison, Kathryn M. Kingsmore, Christian Contarino, Suna Onengut-Gumuscu, Emily Farber, Daniel Raper, Kenneth E. Viar, Romie D. Powell, Wendy Baker, Nisha Dabhi, Robin Bai, Rui Cao, Song Hu, Stephen S. Rich, Jennifer M. Munson, M. Beatriz Lopes, Christopher C. Overall, Scott T. Acton \& Jonathan Kipnis

Correction to: Nature https://doi.org/10.1038/s41586-018-0368-8, published online 25 July 2018.

In Fig. 2 of this Article, owing to an error during the production process, the panel labels ' $n$ ' and 'o' overlapped the figure. In addition, the HTML version of Extended Data Fig. 9 was incorrectly showing as Fig. 2. These errors have been corrected online. 